\title{
Investigating the Success Rate of Sex Selection in Cycles of Intrauterine Insemination of Sperm Using an Albumin Gradient Method in Infertile Couples Referring to the Omid Persian Gulf Infertility Center of Bushehr, Iran
}

\author{
Shahnaz Ahmadi ${ }^{1}$, Elham Rahmani ${ }^{2}$, Niloofar Motamed ${ }^{3,4}$, Fatemeh Sadeghi ${ }^{5}$
}

\begin{abstract}
Objectives: Sex selection is an important concern for some couples. One of the relatively more simple and inexpensive methods, which does not need to use toxic chemicals to separate the $\mathrm{Y}$ and $\mathrm{X}$ sperms, is the Ericsson method. Therefore, the aim of this study is to evaluate the results of sex selection in infertile couples by using the albumin gradient method.

Materials and Methods: The present study is a quasi-experimental clinical trial study, conducted on 80 infertile couples. After the induction of ovulation and a vaginal sonography, sperm samples were prepared using the albumin gradient method, after which intrauterine insemination (IUI) was conducted. The rate of successful sex selection was then calculated.

Results: Among 80 cases of performed IUI, 22 cases (27.5\%) were successful, among which 3 cases (8.3\%) suffered miscarriages and 19 cases $(23.7 \%)$ were associated with a successful pregnancy. The success rate of sex selection was $59.1 \%$ in successful pregnancies. In general, the sex selection success rate was higher for the male gender; as a result, the success rates in 68 requests for male sex and 12 requests for female sex totaled 12 cases $(17.6 \%)$ and 1 case $(3.8 \%)$, respectively.

Conclusion: Albumin gradient is an appropriate sex determination method in IUI cycles due to a $23.7 \%$ chance of pregnancy in infertile women, while the success rate is $59.1 \%$ in sex determination.

Keywords: Infertility, Insemination, Sex preselection, Spermatozoa
\end{abstract}

\section{Introduction}

Failure of a woman to become pregnant during one year of intercourse without the use of contraceptive methods is called infertility (1). Researchers believe that infertility has become one of the major social problems in the society, today. However, infertility is one of the areas in the medical field that can help cure this problem using data from relevant researches $(2,3)$.

Infertility is considered as one of the most bitter and stressful life experiences for young couples because of the many psychiatric symptoms that occur during the diagnosis and treatment process. These are in addition to the physical problems, which disrupt their way of life and the consequent problems they experience in their relationships with their spouses, family members, friends and colleagues (4-12). Sometimes, psychological impacts of infertility may also affect the cooperation between patients, the medical team and medical staff.

On the other hand, an increased infertility rate sometimes leads couples to select a specific gender (13). Sometimes, couples are not always infertile, but certain defects are present in one gender of their children, so they have no option but to seek gender determination methods. Moreover, sometimes the interest of some fertile couples in a specific gender has led them to determine their child's gender. In the older days, when a male workforce was more important than the female workforce, if a family had no son, the number of the family members could be increased beyond eight in order to achieve the blessing of a son. That is why the statistics of children of a family were high. Also, many families suffered severely because of this issue. There is also often disagreement between spouses in newly established families over a child's gender; some prefer sons and some prefer daughters. The proven/ established methods of determining a child's gender is a very complicated phenomenon beyond our imaginations.

Today, by using medical, biology and nutritional sciences, it has been has shown that parents can select their child's gender. It should be noted that all of these methods are relative and that only a laboratory method is definite (1416). Of course, this method is used more in couples with disabilities in one type of gender of children. 


\section{Materials and Methods}

This study was a semi-experimental clinical trial, which was conducted on infertile couples who wanted to determine the gender of their child and who were referred to the Bushehr Omid Persian Gulf Infertility Center and Abolfazle Clinic from October 2015 to January 2016. All patients signed an informed written consent. The study was approved by the ethical committee of Bushehr University of Medical Science. The inclusion criteria included a patient being aged between 20 and 40 years, the opening of the fallopian tubes on the HSG or laparoscopy, normal semen analysis test, a request to determine the gender and the desire to enter the study. The exclusion criteria included medical illnesses such as thyroid disorders, increased prolactin and other endocrine diseases and uterine problems, such as myoma and polyps. Primary or secondary infertility and years of infertility were not considered effective in this study; although those patients who were eligible for in vitro fertilization (IVF) or intracytoplasmic sperm injection (ICSI) were not included in this study.

After the initial sonography and from the third to the seventh day of menstruation, the patient was instructed to take 2 tablets of letrozole (Femara, Novartis, Canada) orally, every day. On the eighth and ninth days, 75-150 units of Gonal F (Gonal f, Serono, Aubnne, Switzerland) were subcutaneously injected. On the tenth day, a vaginal ultrasonography was done and then ultrasonographies were repeated every other day, depending on the growth of the follicles. When 1-3 follicles of 19-24 mm in size were seen during the transvaginal sonography, 10000 units of HCG (Pregnyl, Organon, Oss, Netherlands) were administered through an intramuscular injection (IM). Semen samples were taken 36 hours after this and the following additional steps were carried out on the samples. Semen samples were collected by using the self-excited method in disposable sterile containers and then kept at a temperature of $37^{\circ} \mathrm{C}$. The status of the seminal fluid macroscopic parameters, including volume, agglutination and consistency became clear by accessing the World Health Organization (WHO). Afterwards, concentration, type and percentage of motility of sperms were determined using a Makler Chamber. Giemsa staining was used to investigate the sperm morphology and concentration of round cells and 100 sperms, along with non-sperm cells that were fragmented among them.

Two to three milliliters of seminal fluid was poured into a Fanconi tube and albumin-free (pre-heated) Hams F10 medium was added in the same value. The mixture was later centrifuged at $2000 \mathrm{rpm}$ for 10 minutes. The supernatant was discarded and $2 \mathrm{~mL}$ of Hams was added into it. Sperms containing $\mathrm{X}$ and $\mathrm{Y}$ chromosomes were separated using albumin gradient in the following manner:

1. Four milliliters of heated human albumin $10 \%$ (at the laboratory temperature) was equally added into four small Falcon tubes, into each of which $0.5 \mathrm{~mL}$ sperm solution prepared beforehand was added and placed for 30 minutes at $37^{\circ} \mathrm{C}$.
2. $0.5 \mathrm{ml}$ of sediment was gently removed from each tube. Due to the presence of albumin $10 \%$ and $37^{\circ} \mathrm{C}$ for 30 minutes, motile sperms were deposited and a sample was taken from the deposited part.

3. Four small Falcon tubes were used and $0.5 \mathrm{~mL} 20 \%$ albumin and then $1 \mathrm{~m}$ of 12.5 albumin was added to each tube. Finally, 0.5 of sperm solution from the previous step was added and maintained for 45 minutes at $37^{\circ} \mathrm{C}$.

Thirty-six hours after the HCG injection and preparation of seminal fluid on the day of intrauterine insemination (IUI), the child's sex was selected for each of the couples. Finally, the success rate of the sex selection by using the albumin gradient technique for sperm separation in IUI cycles was studied. All the information, including the couples' ages, sperm motility, sperm morphology, number of sperm, the expected and actual gender of the fetus and the number of cycles, was collected in the data form and the analysis was carried out.

\section{Results}

Eighteen couples were included in the study. The minimum and maximum age for the women ranged between 20 and 40 years and their average age was $30.95 \pm 5.02$. There was at least one previous pregnancy in $63.3 \%$ of cases and a primary pregnancy only accounted for $36.7 \%$ of cases (Table 1).

A total of $85 \%$ (68 couples) desired to have a son, while $15 \%$ (12 couples) desired to have a daughter, respectively. Among the 80 couples, 58 cases $(72.5 \%)$ experienced an unsuccessful IUI and 22 cases (27.5\%) had a successful IUI, among which 3 cases (8.3\%) suffered abortion and 19 cases $(23.7 \%)$ experienced successful pregnancy (Table 2).

Considering the 22 successful IUI cases (including 3 cases of abortion), the success rate of sex selection was 59.1\%. Among the 19 cases of successful pregnancy, sex selection was successful in 13 cases $(68.4 \%, 95 \% \mathrm{CI}$ : 43.44-87.42) and was unsuccessful in 6 cases (31.6\%).

Table 1. Demographic Of Infertile Couples Referring to Omid Persian Gulf Infertility Center for Sex Selection

\begin{tabular}{lcll}
\hline Variable & Mean \pm SD & Min & Max \\
\hline Age & $30.95 \pm 5.02$ & 19 & 40 \\
Number of previous pregnancies & $1.05 \pm 0.95$ & 0 & 4 \\
Number of IUI cycles & $1.18 \pm 0.41$ & 1 & 3 \\
\hline
\end{tabular}

Table 2. The Results of IUI for Sex Selection in Infertile Couples Referring to Omid Persian Gulf Infertility Center for Sex Selection

\begin{tabular}{lcc}
\hline Variable & Number & Percent \\
\hline Expected boy & 68 & 85 \\
Expected girl & 12 & 15 \\
Obtained boy & 15 & 18.75 \\
Obtained girl & 4 & 5 \\
IUl success & 22 & 27.5 \\
Miscarriage & 3 & 3.8 \\
\hline
\end{tabular}


However, the overall success rate of 13 out of 80 couples, considering both the success rate in conducting IUI and pregnancy success rate, was $16.25 \%$ (95\% CI: 8.24-26.18). In all 80 cases, the success rate was higher for the male gender. As such, in 68 requests for a male gender and 12 requests for a female gender, 12 cases $(17.6 \%)$ and 1 case $(3.8 \%)$ were successfully determined. Also, among 19 successful pregnancies, out of the 15 male-requested cases, $12(80 \%)$ were successful, whereas out of the 4 female-requested cases, 1 (25\%) was successful. There was no significant difference between the two successful and unsuccessful IUI groups in terms of the mean number of previous pregnancies $(P=0.68)$, the percentage of sperm with normal morphology $(P=0.50)$ and the number of cycles of IUI ( $P=0.98$; Table 3$)$. However, the sperm count in successful IUI group was significantly higher than the unsuccessful group $(P=0.035)$.

Considering the level of normal sperm by as much as 40 million, the percentage of sperms counted over 40 million was not significantly different between the two groups $(P=1)$. Also, the two groups in terms of sperm motility $(P=0.34)$ and the number sperms, which moved forward $(P=1)$, was not significantly different (Table 4$)$.

\section{Discussion}

In the present study, which was conducted to investigate the success rate of the albumin gradient method in selecting the sex of the fetus, 80 infertile couples who wanted to determine the sex of their unborn child were entered into the study. Finally, 58 failed cases and 3 cases of abortion from the remaining 22 cases occurred. The sample size in this study was higher than that of other studies, which, in turn, creates a broader and more accurate scope of information. Also, the possibility of errors in this sample size is lower, as it is easier to achieve the desired results. However, in the study conducted by the Center of Hong Kong (17) using the Ericsson method, 18 patients were studied, regardless of the 4 cases that resulted in an abortion; the sample size has been decreased and, in turn, this affects the results obtained. In another study, Nasresfahani et al (18) studied 45 couples and, in another study by Khalili et al (19), the sample size was equal to 32 subjects. Thus, according to the results of various studies, a smaller sample size is effective in achieving the desired results.

In the present study, the letrozole tablet, which is effective in sex determination, has been used for patients on days 3 to 7 of their menstruation period. In his study, Silverman et al (20) used clomiphene citrate during ovulation, which increases the chance of delivering female infants. Lighter sperms (Y sperm) in the albumin gradient technique produces much faster cross-human albumin layers than $\mathrm{X}$ sperms (HAS) and are deposited in test tubes (21). Researchers believe that the albumin gradient technique is a good method because it does not use chemicals to separate sperms and the fetus is not manipulated. On the other hand, many researchers including $(17,21,22)$ confirmed the effectiveness of this method, especially in determining the male gender. Meanwhile, other researchers believed that this method was ineffective $(17,23)$. However, Fugger's study suggests that the health of the baby after delivery has been confirmed by this method (24). In subjects aged between 20 to 40 years, the results showed that there was no significant difference between successful and unsuccessful groups, which was not referred to in other studies. Other findings that showed no significant differences between the successful and unsuccessful groups included the percentage of sperm with normal morphology. The number of cycles and sperm count was the only factor that was different in the two groups in this study and was not mentioned in other studies.

The above-mentioned factors suggest the accuracy and extent of our study, which are indicative of the superiority of our study compared with other studies. Another factor, which was investigated in the present study, was

Table 3. The Comparison of 2 Groups of Patients in Infertile Couples Referring to Omid Persian Gulf Infertility Center for Sex Selection

\begin{tabular}{|c|c|c|c|c|}
\hline Variable & $\begin{array}{l}\text { IUI Successful } \\
\text { (Mean } \pm \text { SD) }\end{array}$ & $\begin{array}{c}\text { IUI Unsuccessful } \\
\text { (Mean } \pm \text { SD) }\end{array}$ & $t / z$ & $P$ Value \\
\hline Age & $31.31 \pm 3.35$ & $30.88 \pm 5.29$ & -0.27 & 0.78 \\
\hline Number of previous pregnancy & $0.92 \pm 0.86$ & $1.07 \pm 0.97$ & -0.41 & 0.68 \\
\hline Number of IUI cycles & $1.23 \pm 0.59$ & $1.16 \pm 0.37$ & -0.02 & 0.98 \\
\hline
\end{tabular}

Table 4. The Comparison Of the 2 Groups of Patients Regarding Quality Indicators of Sperm Analysis Infertile Couples Referring to Omid Persian Gulf Infertility Center for Sex Selection

\begin{tabular}{llccc}
\hline Variable & & Successful Group & Unsuccessful Group & $\chi^{2}$ \\
\hline \multirow{2}{*}{ Sperm count } & Normal & $12(16.2 \%)$ & $62(83.8 \%)$ & $\boldsymbol{P}^{2}$ Value \\
& Abnormal & $1(16.7 \%)$ & $5(83.3 \%)$ & - \\
\multirow{2}{*}{ Sperm motility } & Normal & $13(18.1 \%)$ & $59(81.9 \%)$ & - \\
& Abnormal & 0 & 8 & 0.34 \\
\multirow{2}{*}{ Forward movement } & Normal & $1(16.5 \%)$ & $66(83.5 \%)$ & - \\
\hline
\end{tabular}

a Fisher exact test. 
the history and number of pregnancies, which was not referred to in other studies, except in the study of Khalili et al (19), and this factor is also important in achieving the desired result. The reproductive ability was another factor that was referred to only in the study by Nasresfahani et al (18), who showed that $80 \%$ and $54.4 \%$ of infertile and fertile women could achieve their desired results. Among the factors that were studied include the rate of successful pregnancy, which was reported to be $21.8 \%$ and $16 \%$ in the studies of Khalili et al (19) and Al-Dujaily and Al-Dahan (25), respectively. However, the same rate was $23.7 \%$ in our study, which is higher than the amount in the abovementioned researches.

The last factor that was analyzed here was the success rate of sex determination, regardless of IUI failure and abortion cases. In the study of Silverman et al, the female birth rate was $74 \%(20)$, which is very similar to that of Kalfoglou et al (26). In a study by Nasresfahani, male birth rates in infertile and fertile couples were 54.4\% and $80 \%$, respectively (18); in the study of Khalili et al, however, the success rate of achieving male deliveries was recorded at $71.4 \%$ (19). In a study by a Hong Kong study center, the success rate of achieving a male sex was $80 \%$, which is equal to the rate reported by fertile couples in Nasresfahani et al (18) and the Hong Kong study (17). In Kalfoglou et al reported that the male and female birth rate was $78 \%$ and $55 \%$, respectively (26). However, Al-Dujaily and Al-Dahan reported $76.64 \%$ and $23.36 \%$ as birth rates for male and female genders, respectively (25). The results of the present study showed that the achievement to male and female genders was $80 \%$ and $25 \%$, respectively, which was similar to the results obtained by Al-Dujaily and AlDahan (25).

\section{Conclusion}

The albumin gradient is an appropriate, affordable sex determination method with few side effects in IUI cycles. This is due to a $23.7 \%$ chance of pregnancy in infertile women and the probability of success of $59.1 \%$ in sex determination.

\section{Ethical Issues}

All patients signed an informed written consent. The study was approved by the ethical committee of Bushehr University of Medical Science with ethical code: IRCT201604264339N13.

\section{Conflict of Interests}

None.

\section{Financial Support}

This study was supported by Bushehr University of Medical Sciences.

\section{Acknowledgments}

The authors are grateful to the Bushehr University of Medical Sciences, Vice chancellery of Research \& technology affairs, that approved the proposal and financial support and Bushehr Omid Persian Gulf infertility clinic staff (Mrs. Yazdanikhah, Miss Kiamarci, Mrs. Bakhtiari for data collection). This data was collected from Miss Sadeghi's thesis.

\section{References}

1. Ott MA, Sucato GS, Braverman PK, et al. Contraception for adolescents. Pediatrics. 2014;134(4):e1257-e1281. doi: 10.1542/peds.2014-2300.

2. Eftekhar M, Rahmani E, Pourmasumi S. Evaluation of clinical factors influencing pregnancy rate in frozen embryo transfer. Iran J Reprod Med. 2014;12(7):513-518.

3. Speroff L, Fritz MA, eds. Clinical Gynecologic Endocrinology and Infertility. Philadelphia: Lippincott Williams \& Wilkins; 2005.

4. Aflatoonian A, Rahmani E, Rahsepar M. Assessing the efficacy of aspiration and ethanol injection in recurrent endometrioma before IVF cycle: a randomized clinical trial. Iran J Reprod Med. 2013;11(3):179.

5. Davar R, Rahsepar M, Rahmani E. A comparative study of luteal estradiol pre-treatment in GnRH antagonist protocols and in micro dose flare protocols for poor-responding patients. Arch Gynecol Obstet 2013;287(1):149-153. doi:10.1007/s00404-012-2522-0.

6. Eftekhar M, Firouzabadi RD, Karimi H, Rahmani E. Outcome of cryopreserved-thawed embryo transfer in the $\mathrm{GnRH}$ agonist versus antagonist protocol. Iran J Reprod Med. 2012;10(4):297.

7. Eftekhar M, Rahmani E, Eftekhar T. Effect of adding human chorionic gonadotropin to the endometrial preparation protocol in frozen embryo transfer cycles. Int J Fertil Steril. 2012;6(3):175-178.

8. Eftekhar M, Rahmani E, Mohammadian F. Comparison of pregnancy outcome in half-dose Triptorelin and short-acting Decapeptyl in long protocol in ART cycles: a randomized clinical trial. Iran J Reprod Med. 2013;11(2):133-138.

9. Eftekhar M, Rahsepar M, Rahmani E. Effect of progesterone supplementation on natural frozen-thawed embryo transfer cycles: a randomized controlled trial. Int J Fertil Steril. 2013;7(1):13-20.

10. Firouzabadi RD, Ahmadi S, Oskouian H, Davar R. Comparing GnRH agonist long protocol and gnrh antagonist protocol in outcome the first cycle of ART. Arch Gynecol Obstet. 2010;281(1):81-85. doi:10.1007/s00404009-1073-5.

11. Firouzabadi RD, Rahmani E, Rahsepar M, Firouzabadi MM. Value of follicular fluid vitamin D in predicting the pregnancy rate in an IVF program. Arch Gynecol Obstet. 2014;289(1):201-206. doi:10.1007/s00404-013-2959-9.

12. Rahmani E, Ahmadi S, Motamed N, Yazdani N. Study of association between ovarian volume with the number of antral follicles and third day of menstruation FSH in infertile patients referred to Omid Persian gulf infertility Clinic. Iran South Med J. 2016;19(4):608-619.

13. Schulman JD, Karabinus DS. Scientific aspects of preconception gender selection. Reprod Biomed Online. 2005;10 Suppl 1:111-115. doi:10.1016/S14726483(10)62217-1

14. Kvarnemo C, Simmons LW. Polyandry as a mediator of sexual selection before and after mating. Philos Trans R Soc Lond B Biol Sci. 2013;368(1613):20120042. doi:10.1098/ rstb.2012.0042.

15. Eftekhar M, Khalili MA, Rahmani E. The efficacy of 
recombinant versus urinary HCG in ART outcome. Iran J Reprod Med. 2012;10(6):543-548.

16. Tajbakhsh S, Esfahani MN, Emaneini M, Motamed N, Rahmani E, Gharibi S. Identification of Streptococcus agalactiae by fluorescent in situ hybridization compared to culturing and the determination of prevalence of Streptococcus agalactiae colonization among pregnant women in Bushehr, Iran. BMC Infect Dis. 2013;13(1):420. doi:10.1186/1471-2334-13-420.

17. Milliez J. Sex selection for non-medical purposes. Reprod Biomed Online. 2007;14:114-117. doi:10.1016/S14726483(10)60742-0.

18. Naseresfahani MH, Ahmadi SM, Kalantari SAA, Safaeian AAR, Shahsanai V, Saberali SM. A report on efficiency of albumin gradient aliong with intra uterine insemination (IVI) for gender selection in fertile and infertile couples. Isfahan Med Sch. 2003;21:24-28.

19. Khalili M, Khani B, Baghazadeh S, Tabibnejad N. Sex selection by using albumin gradient technique for sperm separation in IUI cycles. J Reprod Infertil. 2007;8(3):213221.

20. Silverman AY, Stephens SR, Drouin MT, Zack RG, Osborne J, Ericsson SA. Female sex selection using clomiphene citrate and albumin separation of human sperm. Hum Reprod.
2002;17(5):1254-1256. doi:10.1093/humrep/17.5.1254

21. Garner DL, Seidel GE Jr. History of commercializing sexed semen for cattle. Theriogenology. 2008;69(7):886-895. doi: $10.1016 /$ j.theriogenology.

22. Karabinus DS, Marazzo DP, Stern HJ, et al. The effectiveness of flow cytometric sorting of human sperm $\left(\right.$ MicroSort $^{\circledR}$ ) for influencing a child's sex. Reprod Biol Endocrinol. 2014;12:106. doi:10.1186/1477-7827-12-106.

23. Baheri S, Saki G, Asl JM, Khodadadi A. Assessment of the separation of $\mathrm{X}$-and $\mathrm{Y}$-chromosome bearing sperms of fertile men by albumin gradients using real time PCR. Jentashapir J Health Res. 2016;7(2):e31079 . doi:10.17795/ jjhr-31079.

24. Fugger EF. Clinical experience with flow cytometric separation of human X-and Y-chromosome bearing sperm. Theriogenology. 1999;52(8):1435-1440. doi:10.1016/S0093691X(99)00228-9.

25. Al-Dujaily SS, Al-Dahan SE. Gender selection by Ericsson method in intrauterine insemination for infertile couples. Iraqi J Med Sci. 2014;12(1):25-30.

26. Kalfoglou A, Scott J, Hudson K. Attitudes about preconception sex selection: a focus group study with Americans. Hum Reprod. 2008;23(12):2731-2736. doi:10.1093/humrep/den329.

Copyright $\odot 2017$ The Author (s); This is an open-access article distributed under the terms of the Creative Commons Attribution License (http://creativecommons.org/licenses/by/4.0), which permits unrestricted use, distribution, and reproduction in any medium, provided the original work is properly cited. 